\title{
Identifying prodromal symptomology in women who experienced postpartum psychosis: a grounded research study
}

\begin{abstract}
Objective: The aim of this study was to identify prodromal postpartum psychosis symptoms that are experienced by women prior to the diagnosis of postpartum psychosis.

Methods: A qualitative, retrospective approach was utilized using grounded theory to conduct interviews with 13 women who had a history of postpartum psychosis. The interviews were transcribed and identification of categories, properties, and iterative hypothesis development and testing through the use of constant comparative analysis was performed. Demographic variables including age, marital, status, level of education, gravity, parity, and a history of mental health diagnosis and family history of mental illness were obtained.
\end{abstract}

Results: Participants identified symptoms that fell within four major core themed groups. Participants identified the following themes in the period prior to diagnosis of postpartum psychosis: Sleep disturbances were characterized as an inability to sleep or no desire to sleep. Significant mood changes were categorized as either excitement or hyperactivity or anxiety or depression. Alteration in thought processes were expressed as being in a fog and having racing thoughts. Psychological stress was identified by the participants as feeling crazy and being completely paranoid. A conceptual model naturally emerged from the core categories identified in the study.

Conclusion: Four core categories of prodromal postpartum psychosis symptoms were identified. Practitioners who care for pregnant women should assess for risk factor for postpartum mood and anxiety disorders when performing perinatal depression screenings. Women at risk should undergo additional monitoring in the postpartum period for prodromal symptoms of postpartum psychosis. Education of prenatal practitioners can help increase awareness of early symptoms of postpartum psychosis.
Volume 2 Issue 6 - 2017

\author{
Michele R Davidson,' McClain Samspon, ${ }^{2}$ \\ Nathan S Davidson ${ }^{3}$ \\ 'George Mason University, USA \\ 2University of Houston, USA \\ ${ }^{3}$ Be Well Medical Center, USA
}

Correspondence: Michele R Davidson, School of Nursing, George Mason University, 416 Brougham Ct, Chesapeake,VA 23322, USA, Tel I-703-298-3247, Email mdavidso@gmu.edu

Received: April 07, 2017 | Published: October 03, 2017

Keywords: postpartum depression, postpartum, postpartum mood and anxiety disorders, perinatal depression, postpartum depression

\begin{abstract}
Abbreviations: PPP, postpartum psychosis; PPD, postpartum depression; BPD, bipolar disorder; MDD, major depressive disorder; ACOG, american college of obstetrics \& gynecology; PMADs, postpartum mood and anxiety disorders
\end{abstract}

\section{Introduction}

Alterations in maternal mental health and their effects during pregnancy and in the postpartum period are not uncommon. There is a growing body of evidence that many women with postpartum depression (PPD) actually have actually experienced perinatal depression or depression in pregnancy. It is estimated that up to $15 \%$ of pregnant women experience perinatal depression during pregnancy, while $20 \%$ will experience PPD. ${ }^{1}$ Recently, the American College of Obstetricians and Gynecologists (ACOG) provided recommendations for perinatal screening at least one time during pregnancy using a standardized, validated tool. ${ }^{2}$ The United States Public Health Task Force has also released an evidence summary that advocates for the screening and treatment of perinatal and postpartum depression. ${ }^{3}$ Postpartum mood and anxiety disorders (PMAD) constitute the highest percentage of all postpartum complications in the first year following birth. ${ }^{3}$ Despite the high incidence of maternal health disorders that occur in the postpartum period, many providers do not routinely screen for PMADs. Currently, in the United States, one state, New Jersey, has legislation in place that mandates postpartum depression screening. 2,3

While PPD is often seen and identified in women who have given birth, postpartum psychosis (PPP) is relatively rare, affecting only $0.1 \%$ to $0.2 \%$ of all women who have given birth in the past 12 months. Despite its rarity, the need for early identification of prodromal symptoms that occur prior to diagnosis could significantly reduce the significant morbidity and mortality associated with the disorder., ${ }^{4,5}$ Although uncommon, PPP has been associated with infanticide (2\%), homicide $(2 \%)$, and suicidal acts $(4 \%) .{ }^{5}$ To date, there are no clinical assessment tools to detect the prodromal symptoms related to PPP. As a result, practitioners have little guidance for how to evaluate or screen a woman for symptoms or risk factors that may suggest an impending psychotic episode. While most clinicians can identify an acute psychotic episode, it would be optimal to detect prodromal symptoms - that would allow for immediate referral and treatment before an acute episode unfolds.

This study was designed to identify the presence of symptoms that characterize the prodromal symptomology associated with 
PPP. This study examines the experiences of women who have experienced postpartum psychosis, providing an important dimension to understanding this complex disorder. While recollections from women who suffered the disorder could potentially be perceived inaccurately, they offer an important perspective that identifies how the woman perceives herself during this acute period. This is of value because practitioners and family members only have the signs they objectively observe to report. It is not uncommon for women experiencing early prodromal and psychotic symptoms to withhold information out of fear or due to their alterations with reality. Gaining insight into their perceptions and experiences can assist practitioners in understanding prodromal symptoms that develop before the onset that warrants a diagnosis of PPP.

\section{Research question}

This study was designed to answer the following research question "What symptoms do women exhibit in the prodromal period prior to the diagnosis of PPP?

\section{Background \& significance}

Postpartum psychosis (PPP) is a rare postpartum mood disorder that occurs in only $1-2 \%$ of postpartum women within the first year following childbirth. Postpartum psychosis typically has a rapid onset of symptoms of mania as evidenced by high mood, racing thoughts, depression, rapidly changing mood, and severe confusion, loss of inhibition, paranoia, hallucinations and delusions. The most severe symptoms last from 2 to 12 weeks, and recovery takes 6 months to a year or more. ${ }^{6}$ Although most women develop symptoms during the first 4weeks, there is also a smaller number of women who develop the disorder at the time of weaning or during the first 12 months after birth. ${ }^{6,7}$ Though infrequent, the consequences of experiencing an acute psychotic episode are serious for the woman, her infant, and her family. Many clinicians have not seen an actual case of PPP and may be unfamiliar with its signs and symptoms. The need for screening of risk factors in pregnancy is imperative to identify women at high risk for developing PPP. Risk factors for PPP include a history of mood disorder (bipolar disorder [BPD] or depression), schizoaffective disorder, postpartum depression, postpartum psychosis in a previous pregnancy, and to a lesser extent, schizophrenia. ${ }^{4,5} 8 \mathrm{PPP}$ is 100 times higher in women with pre-existing diagnosis of bipolar disorder. ${ }^{5}$ PPP should be suspected in any patient presenting with significant mood alterations or mania or a previous history of missed or misdiagnosed mood episodes, or a family history of bipolar disorder or PPP. Women with both BPD and a family history of PPP have a 74\% risk of developing PPP. ${ }^{8}$

Women with PPP present with a variety of signs and symptoms including mood lability, distractibility, confusion impaired sensorium, bizarre quality of delusions, and memory loss. ${ }^{8}$ Wisner and colleagues examined signs and symptoms in women with PPP and found a high level of cognitive disorganization including cognitive impairment, bizarre behavior, thought disorganization, lack of insight, delusions of reference, delusions of persecution, and greater levels of homicidal ideation and behaviors. ${ }^{9}$ Additionally, acute episodes of PPP included visual, tactile, and olfactory hallucinations and a delirium-like state. Chandra and colleagues identified that $53 \%$ of women with PPP had delusions that focused on their infants. ${ }^{10}$ This is important, as PPP can negatively impact family functioning, impair maternal-infant attachment, and in some cases, pose a risk of infanticide.
Gaining insight into the woman's perception of her reality as she understood it at the time of the initial symptoms is an important component to understanding the broader aspects of the disorder. Because of the stigma associated with PPP, fear of spousal rejection, strong opposition to hospitalization that would separate the mother from her infant, desire to continue breastfeeding, and fear of legal ramifications related to custody, and determinations of fitness of being a mother, many women hide their symptoms from their family and are reluctant to seek appropriate medical attention.

Researchers have identified symptoms during acute psychotic episodes in women with PPP. Few reports have examined the woman's experiences, or the presence of prodromal symptoms in women who were later diagnosed with PPP. Identification of these women may alert a clinician to an impending psychotic episode. Hebron and colleagues identified prodromal symptoms that were present in women with PPP onset that occurred within the first month following birth. ${ }^{11}$

\section{Materials \& methods}

In this qualitative study, a grounded theory approach was employed to uncover the characteristics and process of women's experiences with symptomology prior to experiencing an acute postpartum psychotic episode. Using grounded theory strategies as described by Corbin and Strauss individual interviews were used to develop credible descriptions of the experiences of the participants before their acute psychotic episode. ${ }^{12}$ The grounded theory approach is appropriate as it focuses on the identification of categories, properties, and iterative hypothesis development and testing through the use of constant comparative analysis. Trustworthiness will be established through the use of bracketing, while credibility will be established by participant checking, where the women will review the researcher's interpretations for accuracy of content and context. Trustworthiness and credibility are necessary components of qualitative research designs. ${ }^{13}$

Women, aged 18years or more, who have experienced PPP, severe PPD with psychotic features, bipolar disorder (BPD) with psychotic features, or major depressive disorder (MDD) with psychotic features within the first 12 months after childbirth were considered for participation. Women meeting eligibility obtained medical clearance from her provider before participating in the study. Potential participants were recruited from provider offices, social service offices, and social media sites. Once women contacted the principal investigator, determination of eligibility criteria was determined. Data collection continued until the researchers determined that theoretical data saturation had occurred. ${ }^{13}$ Based upon similar studies in the literature, it is estimated that the sample size for this study would be 8-10 women.

The interviewer conducted semi-structured interviews with individual women either face-to-face or via the telephone (Appendix A). The interviews were reviewed and coded by two members of the research team with knowledge of postpartum mood disorders. The researchers initially viewed/listened to the interviews individually and used open coding to identify initial words, phrases, or data segments pertinent to the study question. Subsequently, the researchers met collectively to compare their coding and proceeded to identify relevant concepts, dimensions, and categories that characterized the symptoms and prodromal experience of the participants. A constant comparative approach was used to identify relationships, contingencies, and commonalities or variations within, and across, the interviews. Data 
collection continued until data saturation was reached. The grounded theory approach using techniques outlined by Cobrin \& Strauss ${ }^{12}$ was used to gain succinct, though representative, theoretical explanation of the symptoms and process by which the women experienced in the prodromal period before an acute episode of PPP. The analysis revealed important aspects of the women's' experiences that are germane to the research goal of developing an assessment tool that can be effective for screening women in clinical settings.

The risks and benefits of the research were discussed and a consent form was signed. Potential risks of this study included emotional distress caused by reintroduction of unpleasant psychological symptoms that occurred in the past. Possible benefits included an increase in self-awareness, catharsis, and a sense of helping others while allowing researchers to learn from their past experiences. Women were ensured that confidentiality would be maintained and advised that researchers have signed confidentiality statements. This research was submitted and approved by the Institutional Review Board and Office of Sponsored Programs at the Principal Investigator's educational institution.

\section{Results \& discussion}

Participant's demographic data is presented in Table 1. Thirteen participants with a mean age of 29.38years with a range from 19-42years old at the time of the PPP episode were included. Onset of symptoms ranged from 2 days after delivery to 5 months postpartum. All of the women had completed high school; two of the women had associate degrees, four women had completed bachelor degrees, one woman was in her fourth year of college, and one woman had a graduate degree. The majority of the women were married $(n=8)$, while 4 women were single, and one woman reported being separated. Of the three single women, only one did not have a partner. Ten of the women were Caucasian, two were African American, and one woman noted her ethnicity of being mixed race. Of the participants, 7 had a previous mental health diagnosis (5 BPD, one with Major Depressive Disorder (MDD), one with anxiety, and one had a previous history of postpartum depression (PPD). Additionally, 6 of the woman had a first degree relative with mental illness, and an additional two had a second degree relative.

Table I Demographic Information of Participants

\begin{tabular}{|c|c|c|c|c|c|c|c|c|}
\hline $\begin{array}{l}\text { Age at } \\
\text { Birth/ } \\
\text { PPP }\end{array}$ & Gravity & Parity & Ethnicity & $\begin{array}{l}\text { Education } \\
\text { level }\end{array}$ & $\begin{array}{l}\text { Marital/ } \\
\text { Relationship } \\
\text { status }\end{array}$ & $\begin{array}{l}\text { History of } \\
\text { mental health } \\
\text { disorder(MHD) }\end{array}$ & $\begin{array}{l}\text { Family history } \\
\text { of mental health } \\
\text { disorder(FxMHD) }\end{array}$ & $\begin{array}{l}\text { Onset of } \\
\text { prodromal } \\
\text { symptoms }\end{array}$ \\
\hline 24 & 1 & 1 & Cauc & AAS & Married & BPD I & $\begin{array}{l}\text { Aunt Bipolar } \\
\text { Disorder I (BPDI) }\end{array}$ & 2 days \\
\hline 34 & 3 & 2 & Cauc & $\mathrm{HS}$ & Married & None & None & 6 months \\
\hline 38 & 4 & 5 & $\begin{array}{l}\text { African } \\
\text { American }\end{array}$ & HS Diploma & Single & $\begin{array}{l}\text { Postpartum } \\
\text { Depression } \\
\text { (PPD) }\end{array}$ & Sister PPD & 4 months \\
\hline 32 & I & 1 & Cauc & BA & Married & BPD II & Mother PPP & 4 days \\
\hline 22 & 1 & 1 & Cauc & $\begin{array}{l}\text { 4th year of } \\
\text { college }\end{array}$ & Married & None & MGM BPD I & 16 days \\
\hline 42 & 3 & 1 & Cauc & $\begin{array}{l}\text { Graduate } \\
\text { degree }\end{array}$ & Married & None & $\begin{array}{l}\text { Brother Bipolar } \\
\text { Disorder II }\end{array}$ & 5 days \\
\hline 28 & 2 & 1 & Cauc & BS & Married & BPD II & None & 22 days \\
\hline 28 & 4 & 2 & Mixed & AAS & Single & MDD & Sister MDD & II months \\
\hline 35 & 2 & I & Cauc & $\mathrm{BA}$ & Seperated & BPD I & None & 19 days \\
\hline 25 & I & 1 & $\begin{array}{l}\text { African } \\
\text { American }\end{array}$ & $\mathrm{HS}$ & Single & BPD II & None & 7 days \\
\hline 29 & 2 & 2 & Cauc & $\mathrm{HS}$ & Married & None & Adopted & 3 months \\
\hline 26 & 2 & 2 & Cauc & BS & Married & None & Mother MDD & 29 days \\
\hline 19 & 3 & 1 & Cauc & $\mathrm{HS}$ & Single & Anxiety & $\begin{array}{l}\text { Mother Substance } \\
\text { Abuse (SA) }\end{array}$ & 5 months \\
\hline
\end{tabular}


While some women were anxious about talking about their experiences, most were eager to share their symptoms and discuss their experiences. Many expressed a desire to help others gain more insight into the disorder itself and voiced a desire for more knowledge to be given to health care providers. One interview occurred in video chat format that resulted in a face to face meeting; all others took place via phone. Interviews lasted from 48-122 minutes.

Our findings for women with a previous history of BPD were consistent with the literature, in that participants experienced symptomology early in the postpartum period and were diagnosed within the first 4weeks following birth. ${ }^{5,8,11}$ However, some of the women with other psychological diagnoses and those with no personal history of a psychiatric diagnosis were diagnosed either within that first month time period, or later, including one participant that was not diagnosed until 11 months following birth. The period of diagnosis in our study ranged from 2 days to 11 months. In our study, this extended period of diagnosis identifies that further research is needed to differentiate between the onsets of prodromal symptomology in women with different types of psychological risk factors.

The core category in this study was the identification of prodromal postpartum symptoms occurring prior to the diagnosis and full onset of symptoms in PPP. All of the participants noted a set of prodromal symptoms prior to the diagnosis of PPP. Major categories included sleep disturbances, alterations in thought processes, significant mood changes, and psychological changes.

\section{Sleep disturbances}

Sleep disturbances included either an inability to sleep or lack of desire to sleep. The women in this study all noted this to be the initial "unusual" sense they had of something being abnormal.

\section{Inability to sleep or no desire to sleep}

All of the women noted that they were unable to sleep. Significant alterations in sleep were the first thing the participants noted. Women described not sleeping at all or were completely unable to sleep. Despite attempting to rest or lay down, often at the urging of family members, all of the women interviewed described the inability to sleep as a major symptom that initially occurred. One woman reinforced this by repeating:

A. I'm not talking about not falling asleep; I was completely unable to sleep at all. I would not sleep, even in the middle of the night. Everyone told me to lay down, take a nap; I couldn't rest, nap, nothing.

B. Another participant noted that she went days without sleep and then would think she had slept, but had dozed off for 35-45minutes. Women noted that even when support was present, they could not and did not sleep much at all.

C. I swear I didn't sleep for four days before I was in the hospital. It was like I could not turn my mind off, thoughts just raced making sleep completely impossible. I don't remember feeling tired, but I remember that even when I laid down, sleep was beyond my grasp!

\section{Another noted similar symptoms}

I couldn't even put my head on the pillow, I was up again. Mama would say, 'Girl you got to sleep when this baby is sleeping', but I couldn't. I even took Benadryl or something and still, I could not fall asleep. That usually knocks me out, but my body was just 'on' and I couldn't stop it.

\section{Alterations in thought processes}

Women noted a substantial degree of cloudiness in their thoughts and thinking. Women noted feeling disoriented, and described alterations in thinking. Racing thoughts were another major theme that created alterations in thought processes.

\section{In a fog}

Women were asked to describe the feelings that first led them to think they were experiencing PPP. The participants all noted some degree of altered thinking and processing that was significantly different than their normal baseline. Women described this as being "cloudy", "foggy" or being in a trance-like state. One noted:

I felt I was in a complete fog, like I was given some surgical drug (anesthesia) and didn't know what was going on around me. Nothing seemed clear. I was talking but none of the conversations made sense.

Another woman recalled:

I felt like I was in a haze, I just really could not understand things clearly or rationally; I didn't understand why I felt like this; nothing had clarity. My husband was telling me things, reminding me of things the nurses had said before we came home, but it was like it wasn't making sense. I knew it wasn't 'normal', but didn't know what was going on, I just knew, "Damn, some things not right here!"

\section{Racing thoughts}

Many women $(\mathrm{n}=8)$ reported racing thoughts including disruptive thoughts that interfered with their ability to think clearly. Women were clearly distressed that they couldn't seem to control their own thinking. One participant noted:

It was like being on a merry-go-round, the thoughts just constantly came repeatedly. No matter how much I tried to watch TV, focus on the baby, talk to someone, or distract myself, I had these thoughts that I couldn't stop! They were so fast too, like they were in a super-fast track, it's hard to explain, they wouldn't stop!

Another women noted the thoughts were constantly making her anxious:

I kept thinking I have to check on the baby, I have to make sure the baby is OK, I must be a bad mother, I haven't checked on the baby, and then I would be up and down, up and down. Checking, rechecking, but I kept thinking I wasn't checking enough.

\section{Significant mood changes}

All of the women noted a marked change in mood prior to the diagnosis of PPP. Excitement, hyperactivity, and elation were commonly encountered $(n=8)$, although some women $(n=5)$ presented with depressive symptoms noting that they felt more irritable, anxious or worried.

\section{Excitement or hyperactivity}

Many of the women in the study noticed a substantial shift in their normal mood and an increase in their level of activity.

I couldn't stop talking; I seemed hyper. At first I thought was 
getting back to normal after having s cesarean section, but it was like I went from park to overdrive in a minute, and it never stopped!

Another noted that her ability to do many things increased:

I was taking care of the baby, cleaning, cooking dinners, dusting, it was like I was I was super woman and I could do it all.

\section{Anxiety or depression}

Another participant noted that her level of anxiety grew to an extreme level and never decreased:

I had some 'normal anxiety' during pregnancy, normal stuff: worrying about my labor, would I be a good Mom, would I need an epidural, concerned I gained too much weight. Then I became a nervous wreck. I was extreme, I worried non-stop about my milk being not enough, even though I had been breastfeeding OK for three weeks. I worried about anything, everything, from starving the baby, to the baby being abducted, to suffocation of my daughter on my breast. Every thought I had was a fear and was constant anxiety.

\section{Psychological stressors}

All of the women noted some type of psychological stressor. All the women perceived some degree of stress or commented on "feeling crazy" or being in an altered state separate from their previous sense of reality.

\section{Feeling Crazy}

Prior to diagnosis, women noted a sense of slipping from a normal state into an altered state where confusion, disorganization of thoughts, and jumbled thinking occurred. Women noted this using terms such as "losing it", "feeling I was becoming crazy", and "slipping out of my world". One woman explained:

I felt like I as a person was shrinking into someplace and becoming someone I did not know. I could see myself bit by bit going crazy. My thinking wasn't right, it was like I was drugged or something. I kept thinking, 'I am crazy, I have gone crazy.'

\section{Another expressed:}

I truly believed I was crazy! I found myself avoiding people, even my close family, so people would not notice. I knew my thoughts, inability to concentrate, I couldn't even plan my day, it was like I was completely nuts, I was crazy, and I didn't want anyone else to notice.

\section{Completely Paranoid}

Womennoted a sense of paranoia prior to the diagnosis of PPP. Al of the women were either paranoid others viewed them as "crazy" or felt others "had something out for them", were judging them, were planning to take their baby away from them, or had some other suspicious thoughts. One woman explained:

I was sure my husband was having an affair with his ex-wife and they were planning on stealing my baby. I wouldn't let him be alone with our daughter, for fear he'd run out the door with her. I began to listen to their phone calls (when they were planning visitation with his older children) and accuse my husband of cheating and planning on kidnapping (Baby's name).

Another woman described being afraid that germs would get on her baby and would proceed to wash his hands, feet, and face off every time they returned from being in public. Women also feared others around them were talking about them or judging them. One noted that: I was sure my mother-in-law was noting every mistake I made. Being my first, I knew she was comparing me with my sisterin-law and telling everyone I had no clue what to do with a baby. It got to the point, I would hide in my bedroom when I nursed or even changed him. I grew to distrust my husband, his parents, everyone! I felt so alone...

\section{Discussion}

Women in this study demonstrated a unique set of behaviors and experiences in the period prior to the diagnosis of PPP. Many of the symptoms aligned with findings from other studies. The Davidson Conceptual Model of Prodromal Postpartum Psychosis Symptomology identifies four prodromal categories that were observed to occur prior to the diagnosis of postpartum psychosis. These categories were then compared to the literature and found to be consistent with other research findings. They included: sleep disturbances, significant mood changes, alteration in thought processes, and psychological stress. Prodromal symptomology has been actively studied in other mental illnesses, such as schizophrenia, psychotic disorders, and bipolar disorders. ${ }^{14-16}$ These studies have produced various conceptual models and screening tools that screen and are used to identify risk for these psychiatric disorders.

It is important to note that these are prodromal symptoms and are different from risk factors. Prodromal symptomology in postpartum psychosis refers to the symptoms that occur prior to the onset of symptoms that warrant the diagnosis of postpartum psychosis. This study specifically addresses symptomology as a separate entity from risk factors. We define risk factors as the presence of a factor(s) that affects an individual and may put them at risk for developing the disorder. We define symptomology as the set of symptoms characteristic of a medical condition or exhibited by a patient. While our study showed a history of a mental health disorder and a family history of mental health disorders in some of our participants that were risk factors associated with the diagnosis of PPP; the focus of our research was to capture symptomology in participants who had had the disorder, despite known risk factors as it was beyond the scope of this study to examine all possible risk factors. We felt that in order to identify symptoms encountered in the postpartum period prior to diagnosis of PPP, we could begin to identify the prodromal symptoms that suggest a potential diagnosis of PPP. By understanding the prodromal symptoms associated with PPP, the clinician can appropriately screen and provide early detection of the onset of the disorder.

Every participant noted her initial symptom of PPP began with sleep disturbances, either an inability to sleep or lack of desire to sleep. Sleep deprivation in the early postpartum period is not unusual; most new mothers experience sleep deprivation or a reduction in mean REM sleep due to newborn needs. Other researchers have identified inability to sleep or the inability to sleep as an early indicator in PPP. ${ }^{17-22}$ The inability or lack of desire to sleep at all, however, is a unique sign that may be an earlier indicator of PPP. Lewis and colleagues have noted the overlap in sleep disruption and circadian systems disruption in individuals with bipolar disorders..$^{18}$ Because serotonin and dopamine are also found to influence both mood, sleep, and circadian rhythms, research has focused on protected sleep as a mechanism of prevention and found a reduction in relapses in the treatment group. Genetic links could also explain why women with a genetic predisposition to 
bipolar may be more sensitive to neurotransmitter changes that occur with sleep disruption. ${ }^{23}$

Protected sleep, refers to the purposeful opportunities for the mother to engage in sleep. This can include nap periods or planned overnight sleep with a caretaker caring for the baby. Women who cannot sleep, even when protected sleep opportunities are provided should be screened for PPP. It is not unusual for women to report to their health care provider's an inability to sleep. Women may be misdiagnosed with postpartum anxiety and given sedatives for insomnia. If a woman does not respond after sedatives are taken and remains unable to sleep, further evaluation is necessary. Both mania and hypomania states are marked by a decreased need to sleep, typically less than 3hours per 24hour period. ${ }^{24}$ Our findings also demonstrate alterations in thought processes that occur, which is a symptom of mania, and often bipolar disorder. Bipolar disorder and family history of PPP are both risk factors for PPP that have been identified in the literature, and were consistent with our findings. ${ }^{4,8,16,25}$

New mothers can report alterations in clarity of thinking after childbirth, especially with the demands of new role development and sleep interruption, but these are typically minor symptoms that do not impair their ability to care for themselves or their infants.4,26 Forgetfulness is quite different from the experiences of cloudiness or alterations in cognitive thinking and processing described by the participants. The women in the study noted an inability to think clearly, and a true sense of fogginess that led to impairment in functioning and cognitive impairment which disrupted their ability to function. Wisner \& colleagues examined women with PPP and found similar alterations in cognitive abilities including a high level of cognitive disorganization including cognitive impairment and thought disorganization. ${ }^{9}$ Heron and colleagues examined women with early PPP and also identified cognitive impairment and disruption in thought processes, a finding that has been found in other studies. $8,17,22$

Women also noted that they were in a fog and noticed the presence of racing and/or unusual thoughts. Most noted that the thoughts they experienced were unusual for them, and not something they would typically experience. The women noted the thoughts were concerning or troubling. Brockington and colleagues found thought pattern disturbances in women diagnosed with PPP were common. ${ }^{8}$ In mania, recurrent ongoing thoughts are described as racing thoughts or a flight of ideas. ${ }^{24}$ Chandra and colleagues also noted the women in their study reported presence of unusual thoughts. ${ }^{10}$ This represents an important factor in the identification of prodromal symptomology because most women will not share these thoughts with others unless asked. Women in our study knew they were "not right" and were alarmed by them. These alterations in cognitive functioning and thought processes were noted to interfere in daily functioning, an important consideration in screening for prodromal symptoms.

Psychological stress was an early prodromal symptom in which the women noted self-perceived psychological stress. We define this as an awareness that something was wrong with their psychological sense of self. Women noted a sense of feeling crazy as evidenced by confusion, disorganized thoughts, and alterations in cognition and thought processes. In this study, the majority of the women $(n=9)$ noted they perceived themselves as crazy. Other studies have shown similar findings, but this concept has not been widely examined in the past. ${ }^{25}$ Paranoia and suspiciousness are known symptoms in women diagnosed with PPP. ${ }^{27,28}$ In our study, it was noted as a prodromal symptom that occurred before the actual diagnosis and the emergence of hallucinations or delusions. Women often have paranoid thoughts about others opinions of how they are caring for their infant. , $, 8,10^{-10}$

Based on our findings and a comprehensive review of the literature, our research led to the development of a conceptual model which can be used by practitioners to identify clusters of symptoms within categories that indicate a woman could be exhibiting symptoms that could lead to a diagnosis of PPP. This is an important development in the field of PMAD because it can be used to promote future research and provide a foundation to develop a PPP risk assessment and prodromal PPP symptomology screening tool.

Limitations of this study were similar to limitations encountered by others using the grounded research methodology. With grounded theory, there is always a risk for the researcher to become too embedded with the content or to have preconceived ideas. The researchers explored different qualitative research approaches and through thoughtful consideration and outside consultation felt that grounded theory had the best fit since the data was allowed to emerge and themes were able to develop or unfold naturally during the data collection process. Purposeful attempts to control for bias included using techniques outlined in the literature to stay open, actively attempt to eliminate preconceptions and allow the data to guide the research process, while being transparent in determining if an emergent fit occurred. ${ }^{29}$ This study is retrospective in nature and involved interviewing women who had a diagnosis of PPP, which is known to alter thoughts and perceptions during the course of the illness. This could impact the accuracy of recall for the participants.

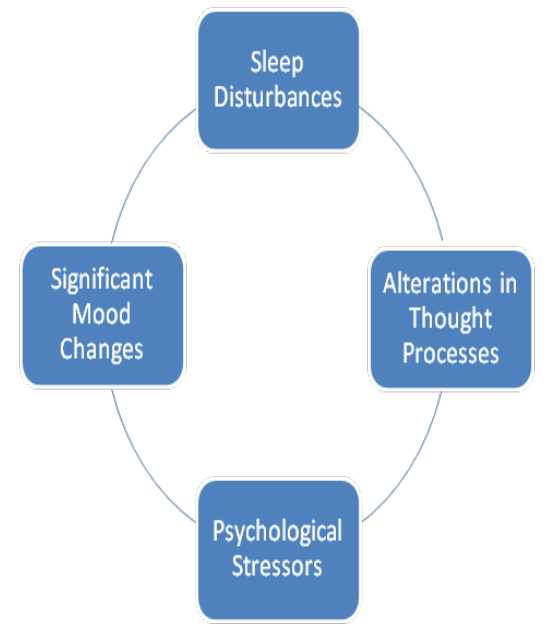

Figure I Davidson Conceptual Model of Prodromal Postpartum Psychosis Symptomology.

\section{Clinical implications}

During pregnancy, practitioners can easily integrate screening for PMAD risk factors into perinatal depression screening. Educating pregnant women and their families, along with practitioners who provide care to women and their new families, can facilitate identification of both early PPD signs and symptoms and the prodromal symptoms associated with PPP. The Davidson Conceptual Model of Prodromal Postpartum Psychosis Symptomology emerged from the data and is a visual representation of the known prodromal risk factors that can be used by practitioners to guide the assessment process. Future research is planned to develop a validated clinical assessment tool that detects risk factors and prodromal symptoms that may indicate a possible postpartum psychosis diagnosis. 
Recommendations for practitioners include a comprehensive review of women's risk factors during pregnancy and identifying possible risk factors for the development of PPP that would warrant an individualized screening approach throughout the postpartum period. The Davidson Conceptual Model of Prodromal Postpartum Psychosis Symptomology can serve as a foundation for postpartum psychosis screening protocols.

\section{Acknowledgements}

The researchers would like to thank Dr. Jeanne Sorrell for her ongoing input and support throughout the research process.

\section{Conflicts of interest}

The authors note no conflict of interest. Dr. Michele Davidson is the Postpartum Psychosis Coordinator, a non-paid, volunteer position for Postpartum Support International, a non-profit organization whose mission is to increase awareness among public and professional communities about the emotional changes that women experience during pregnancy and postpartum.

\section{References}

1. AHRQ. Perinatal depression, prevalence, screening accuracy, and screening outcomes summary. Evidence Report and Technology Assessment, No. 199, Washington, DC, USA; 2005.

2. ACOG. Screening for perinatal depression.ACOG Committee Opinion, No. 630, Washington, DC, USA; 2015.

3. O'Connor E, Rossom RC, Henninger M, et al. Primary care screening for and treatment of depression in pregnant and postpartum women: Evidence report and systematic review for the US Preventive Services Task Force.JAMA. 20I6;3I5(4):388-406

4. Davidson MR. A nurse's guide to women's mental health. New York: Springer; 2012.

5. Spinelli M. Postpartum psychosis: detection of risk and management. Am J Psychiat. 2009; I66(4):405-408.

6. Jones I, Chandra PS, Dazzan P, et al. Bipolar disorder, affective psychosis, and schizophrenia in pregnancy and the post-partum period. Lancet. 20|4;384(9956): I789- I799.

7. Woznicki K. Onset of postpartum depression is more than four-week phenomenon. Medscape. 2005.

8. Brockington IF, Cernik KF, Schofield EM, et al. Puerperal psychosis: Phenomena and diagnosis. Arch Gen Psychiatry. 1981;38(7):829-833.

9. Wisner KL, Peindl K, Hanusa BH. Symptomatology of affective and psychotic illnesses related to childbearing. J Affect Disord. 1994;30(2):77-87.

10. Chandra PS, Bhargavaraman RP, Raghunandan VN, et al. Delusions related to infant and their association with mother-infant interactions in postpartum psychotic disorders. Arch Womens Ment Health. 2006;9(5):285-288.

11. Harlow BL, Vitonis AF, Sparen P, et al. Incidence of hospitalization for postpartum psychotic and bipolar episodes. Arch Gen Psychiatry. 2008;64(I):42-48
12. Cobrin J, Strauss A. Basics of qualitative research:Techniques and procedures for developing grounded theory. Thousand Oaks, CA: Sage Publications, 2008.

13. Jacelon CS, O'Dell KK. Case and grounded theory as qualitative research methods. Urological Nursing. 2005;25(I):49-52.

14. Gourzis P, Katrivanou A, Beratis S. Symptomatology of the initial prodromal phase in schizophrenia. Schizophr Bull. 2002;28(3):4I5-429.

15. Lo Cascio N, Saba R, Hauser M, et al. Attenuated psychotic and basic symptom characteristics in adolescents with ultra-high risk criteria for psychosis, other non-psychotic psychiatric disorders and early-onset psychosis. Eur Child Adolesc Psychiatry. 20 I6;25(I0): I09I-I I 02.

16. Bechdolf A, Ratheesh A, Cotton SM, et al. The predictive validity of bipolar at-risk (prodromal) criteria in help-seeking adolescents and young adults: a prospective study. Bipolar Disorders. 20।4; I6(5):493-504.

17. Heron J, McGuinness M, Blackmore ER, et al. Early postpartum symptoms in puerperal psychosis. BJOG. 2007; I I5(3):348-353.

18. Bergink V, Rasgon N, Wisner KL. Postpartum psychosis: madness, mania, and melancholia in motherhood. American journal of psychiatry. 2016;173(I2): II79- II88.

19. Sharma V, Mazmanian D. Sleep loss and postpartum psychosis. Bipolar Disorders. 2003;5(2):98-105.

20. Strouse TB, Szuba MP, Baxter LR. Response to sleep deprivation in three women with postpartum psychosis.J Clin Psychiatry. 1992;53(6):204-206.

21. Sharma V, Smith A, Khan $M$. The relationship between duration of labour, time of delivery, and puerperal psychosis. J Affective Disord. 2004;83(2-3):215-220.

22. Marks MN,Wieck A, Checkley SA, et al. Contribution of psychological and social factors to psychotic and nonpsychotic relapse after childbirth in women with previous histories of affective disorder. J Affective Disord. 1992;24(4):253-263.

23. Frank E, Kupfer DJ,Thase ME, et al.Two-year outcomes for interpersonal and social rhythm therapy in individuals with bipolar I disorder. Arch Gen Psychiatry. 2005;62(9):996- 1004.

24. APA Diagnostic \& Statistical Manual of Mental Disorders. 5th ed.American Psychological Association. Washington, DC, USA

25. Sit D, Rothschild JA, Wiener KL. A review of postpartum psychosis. J Womens Health. 2006; I5(4):352-365.

26. Ladewig PL, Davidson MR, London ML. Contemporary Maternal and Newborn Nursing. 9th ed. Pearson Health. Boston, USA; 2017.

27. Mighton CE, Inglis AJ, Carrion PB, et al. Perinatal psychosis in mothers with a history of major depressive disorder. Arch Womens Ment Health. $2016 ; 19(2): 253-258$.

28. Hay PJ. Post-partum psychosis: Which women are at highest risk? PLoS Med. 2009;6(2):el000027.

29. Deady R. Reading with methodological perspective bias: A journey into classic grounded theory. Grounded Theory Review. 20 I I; I (I0):22-39. 